\title{
Central low-grade osteosarcoma with pagetoid bone formation: a potential diagnostic pitfall
}

\author{
Alessandro Franchi ${ }^{1}$, Patrizia Bacchini ${ }^{2}$, Carlo Della Rocca ${ }^{3}$ and Franco Bertoni ${ }^{2}$ \\ ${ }^{1}$ Department of Human Pathology and Oncology, University of Florence, Florence, Italy; ${ }^{2}$ Department of \\ Musculoskeletal Oncology, Istituto Ortopedico Rizzoli, Bologna, Italy and ${ }^{3}$ Department of Experimental \\ Medicine and Pathology, La Sapienza University, Rome, Italy
}

\begin{abstract}
Central low-grade osteosarcoma is an uncommon form of osteosarcoma, which is often difficult to distinguish from benign bone lesions. We reviewed the radiographic studies, the histologic material and the clinical records of two patients with central low-grade osteosarcoma that closely simulated the histologic appearance of Paget's disease of bone. The patients were two women aged 46 and 53 years. Radiologically, they presented a large ill-defined densely sclerotic lesion involving the proximal tibia. Both lesions only focally presented the conventional histologic appearance of central low-grade osteosarcoma, with a proliferation of fibroblast-like cells embedded in a dense collagenous stroma and irregular anastomosing tumor bone trabeculae. The most striking feature was the presence of extremely thickened irregular plates of bone with an irregular mosaic pattern of cement lines that closely resembled that of Paget's disease of bone. One patient, who had been initially treated for Paget's disease for 7 years, experienced disease progression. At resection of proximal tibia, there was evidence of dedifferentiation to high-grade osteosarcoma. After 2 months, she developed local recurrence that was treated with above-knee amputation, followed by chemotherapy. She died with multiple lung metastases 4 months later. The other patient is alive 9 months after wide tumor resection. These cases further expand the spectrum of central low-grade osteosarcoma, and underscore the diagnostic difficulties in separating central low-grade osteosarcoma from benign bone diseases, which may lead to delay in diagnosis, inadequate treatment, and eventually to dedifferentiation. Recognition of this variant of central low-grade osteosarcoma is based on the aggressive radiologic appearance and on adequate tumor sampling for histologic examination.
\end{abstract}

Modern Pathology (2004) 17, 288-291, advance online publication, 13 February 2004; doi:10.1038/modpathol.3800069

Keywords: bone; osteosarcoma; central low-grade; Paget's disease

Central low-grade osteosarcoma is an uncommon but well-recognized variant of osteosarcoma, which is characterized by a significantly better prognosis than conventional high-grade osteosarcoma, with slow growth, low metastatic rate and prolonged survival after treatment. ${ }^{1-3}$ Central low-grade osteosarcoma may be easily confused with benign intraosseous lesions, particularly with fibrous dysplasia. The differential diagnosis is based on the aggressive radiographic features of the lesion, as well as on the recognition of an infiltrating growth pattern with permeation of the bone marrow spaces and entrapment of the host trabeculae. These tumors are adequately treated with wide excision, while incomplete resection is often followed by local

Correspondence: Professor Alessandro Franchi, MD, Department of Human Pathology and Oncology, Viale Morgagni 85, 50134 Firenze, Italy.

E-mail: Franchi@unifi.it

Received 10 June 2003; revised 15 October 2003; accepted 16 October 2003; published online 13 February 2004 recurrence, with possible dedifferentiation to highgrade sarcoma, followed by distant dissemination.

In this report, we describe the clinicopathologic findings in two cases of central low-grade osteosarcoma, characterized by a peculiar histologic aspect with extensive bone matrix production and formation of markedly irregular bony trabeculae, simulating the histologic appearance of Paget's disease of bone.

\section{Case reports}

Case No. 1

\section{History}

A 53-year-old woman presented with a 12-month history of left leg weakness and pain, which had been initially related to degenerative intervertebral disc disease. Radiographic examination revealed a purely sclerotic lesion of the proximal tibia (Figure 1a), which involved the metadiaphyseal region. 


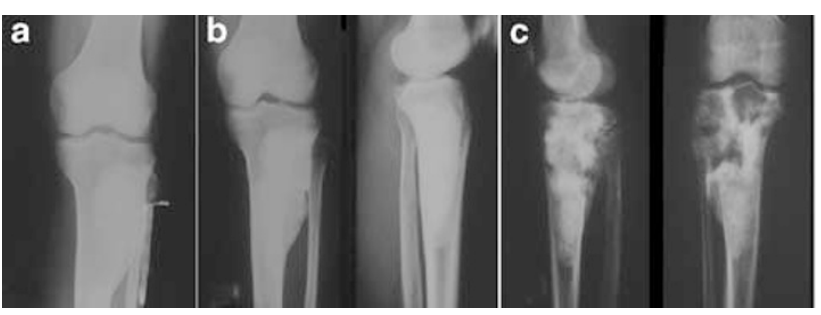

Figure 1 Case 1: (a) Radiograph showing an ill-defined purely sclerotic lesion of the proximal tibia. (b) Radiographs taken after 6 years showed that the lesion had enlarged. (c) After 1 year irregular lytic areas had appeared.

After incisional biopsy, a diagnosis of Paget's disease was made at another institution, and treatment with calcitonin was started. After 6 years, $\mathrm{X}$-ray studies showed that the sclerotic lesion of the proximal tibia had enlarged towards the epiphysis and the diaphysis (Figure 1b). After 1 year, the patient complained of worsening of pain and appearance of swelling in the region of the left proximal tibia. At this time, X-ray studies showed the appearance of irregular lucent areas within the sclerotic lesion (Figure 1c). A new biopsy was performed, which showed a high-grade fibroblastic osteosarcoma. At this time, the original slides of the first biopsy were reviewed, and were reinterpreted as showing low-grade osteosarcoma. She underwent marginal excision of proximal tibia, followed by chemotherapy treatment. After 4 months, multiple local recurrences were detected, which were treated with above-knee amputation. The patient died 2 months later (8 years after clinical presentation), with multiple lung metastases.

\section{Case No. 2}

\section{History}

This 46-year-old woman presented with pain in the right knee region of 12 months duration. Radiographic studies demonstrated an ill-defined densely sclerotic lesion of the proximal tibia, measuring $10 \times 5 \times 4 \mathrm{~cm}$ (Figure 2). After incisional biopsy, the lesion was resected with wide excision. The patient is alive with no evidence of disease 8 months after diagnosis.

\section{Histopathologic findings}

The microscopic features observed in the first biopsy specimen of patient 1 , as well as in the biopsy and resection specimen of patient 2 are very similar, and therefore a combined description is presented. Both lesion were characterized by the presence of extremely thickened, often coalescent plates of bone with an irregular mosaic pattern of cement lines, which closely resembled that of Paget's disease of bone (Figures 3-5). Often, osteoblast-like cells could be identified at the surface of bony trabeculae, together with osteoclast-like cells
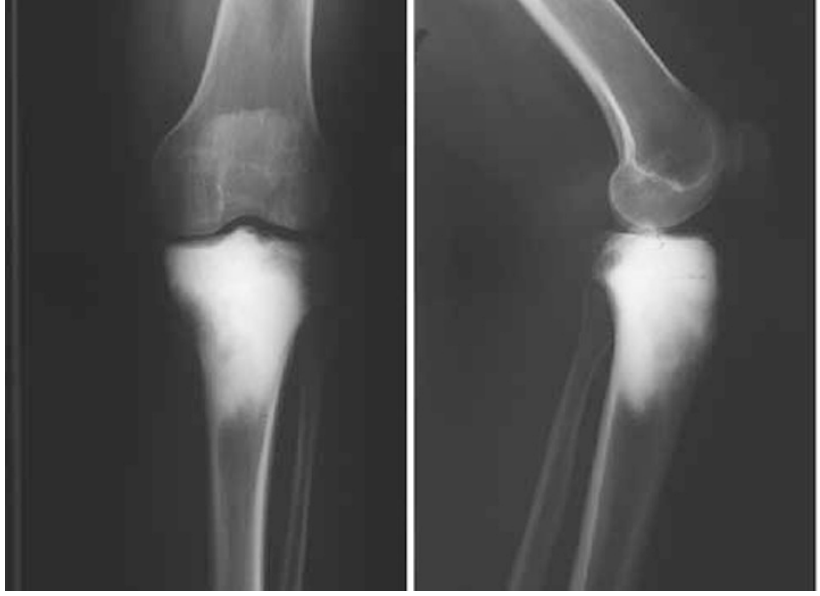

Figure 2 Case 2: anteroposterior and lateral views showing an illdefined densely sclerotic lesion of proximal tibia with cortical invasion.

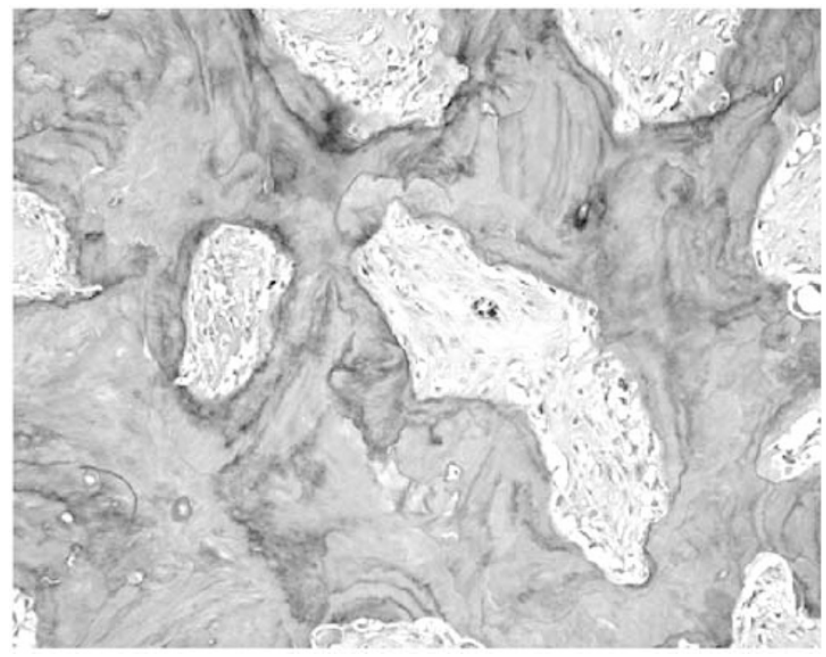

Figure 3 Low-power view showing heavy irregular trabeculae of tumor bone.

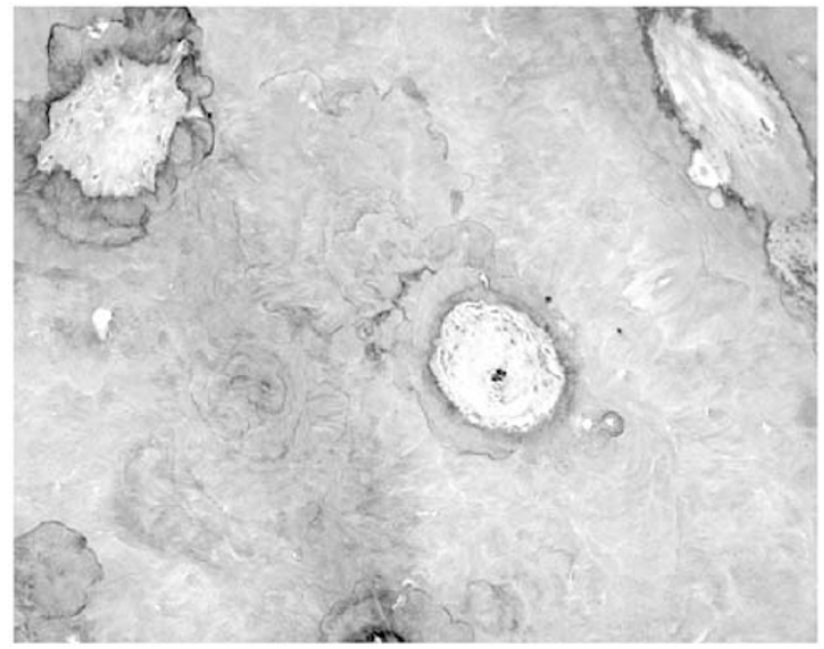

Figure 4 Areas dominated by the formation of solid coalescent plates of tumor bone. 

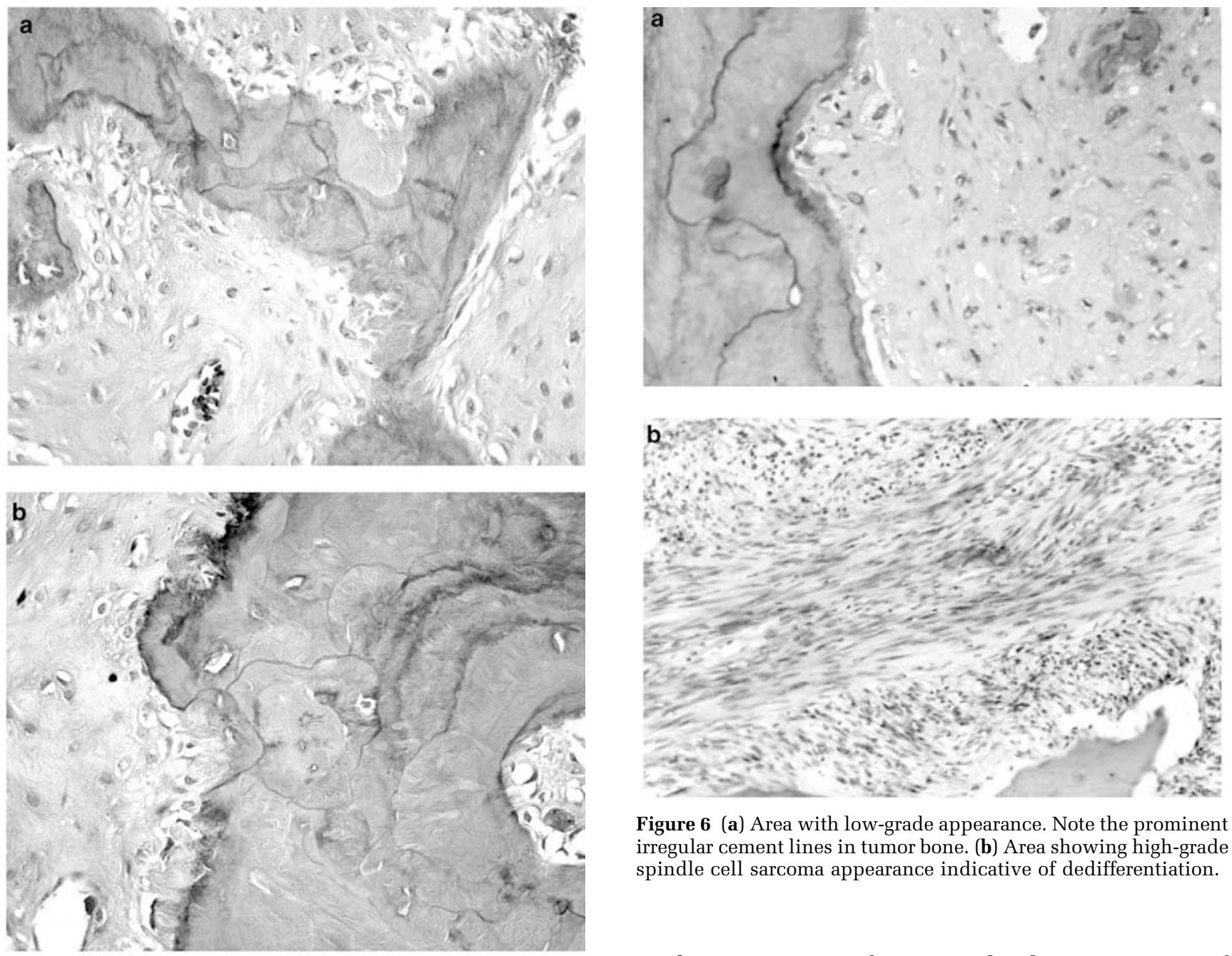

Figure 6 (a) Area with low-grade appearance. Note the prominent irregular cement lines in tumor bone. (b) Area showing high-grade spindle cell sarcoma appearance indicative of dedifferentiation.

Figure 5 (a and b). High-power view showing tumor bone with an irregular mosaic pattern of cement lines resembling that of Paget's disease of bone. Tumor cells are embedded in abundant collagen and show little atypia.

within Howship's lacunae. The intertrabecular space showed scanty spindle or stellate cells with minimal atypia, embedded in abundant collagen matrix. Only in focal areas, the tumors had the appearance of conventional central low-grade osteosarcoma, with bundles of uniform fibroblast-like cells associated with irregular anastomosing tumor bone trabeculae, which infiltrated the medullary spaces with encasement of pre-existing trabeculae. No mitotic figures were identified.

The second biopsy specimen of patient 1 , showed a cellular proliferation of atypical spindle cells, arranged in intersecting bundles, with focal areas of lace-like osteoid formation, consistent with highgrade fibroblastic osteosarcoma. The specimen of proximal tibia resection showed a combination of the two aspects, with focal residual areas of lowgrade osteosarcoma, intermixed with the high-grade fibroblastic osteosarcoma component (Figures 6a and $b)$.

The specimen of proximal tibia resection of patient 2 presented a heavily calcified tumor mass, which diffusely involved the medullary cavity and infiltrated the cortical bone in several points. The specimen was extensively sampled, and the histologic features of the tumor were similar to those of the incisional biopsy. In particular, no areas of highgrade osteosarcoma were identified.

\section{Discussion}

Central low-grade osteosarcoma is a rare variant of osteosarcoma which is characterized histologically by a proliferation of elongated bland spindle cells arranged in interlacing fascicles in heavy collagenous background, with bone or osteoid production. The amount of bone or osteoid produced is variable. In a minority of cases, there is scant osteoid production, and therefore the histologic picture is dominated by the spindle cell proliferation, resulting in aspects mimicking the appearance of desmoplastic fibroma or lowgrade fibrosarcoma. ${ }^{1,2}$ More commonly, the tumor is characterized either by production of heavy bony trabeculae similar to those seen in parosteal 
osteosarcoma, or by thin osteoid seams having the 'Chinese character' appearance seen in fibrous dysplasia. ${ }^{1,2}$ In rare instances, the tumor may mimic the appearance of osteoblastoma, or may have a fibrochondromyxoid aspect, or may show areas of cartilage matrix production. ${ }^{1}$

Instead of the well-recognized conventional patterns, the cases presented here are mainly characterized by very low cellularity and production of extremely dense, sclerotic trabecular bone with an irregular mosaic pattern of cement lines. This appearance closely mimics that of Paget's disease of bone, resembling the histologic pattern of the final phase, the so-called osteoblastic or sclerotic phase. The picture is dominated by bone formation, and the irregularly shaped trabeculae show the characteristic mosaic pattern of cement lines. In this phase, there is relatively little cellular activity, and the marrow spaces are almost entirely filled with dense fibrous tissue containing scattered blood vessels. The differential diagnosis is based on the recognition of aggressive histologic features in central low-grade osteosarcoma, such as permeation of the bone marrow, with entrapment of the host trabeculae, which are usually present at the growing edge of the tumor. However, especially in biopsy material, these features may be absent, and therefore it is essential to evaluate carefully the radiologic aspect, which in both the present cases was indicative of malignancy, for the presence of poorly defined tumor margins, cortical invasion, and extraosseous extension. On the other hand, radiographs did not show the typical features of Paget's disease of bone, which in the initial phase is characterized by lytic lesions, evolving in a mixed lytic and sclerotic phase, and finally in a sclerotic phase with bone deformity, consisting of anterior and lateral bowing of the tibia.

The peculiar pattern of pagetoid bone formation observed in the present cases is probably the result of a very slow growth, with heavy bone matrix production and remodeling, in accordance with the very long clinical history. A similar pattern of bone formation has been previously described in other bone-forming lesions. Okada et $a l^{4}$ identified pagetoid bone formation in 5\% of parosteal osteosarcomas in their series. More recently Riminucci et $a l^{5}$ have described a 'pagetoid' variant of fibrous dysplasia involving the craniofacial bones, characterized by dense sclerotic bone formation with prominent cement lines closely resembling that seen in Paget's disease of bone.

The possibility should be considered that these tumors represent central low-grade osteosarcoma associated with Paget's disease of bone. However, this hypothesis is unlikely because sarcomas arising in Paget's disease, including osteosarcomas, malignant fibrous histiocytomas, fibrosarcomas, chondrosarcomas and mixed forms, are high-grade sarcomas with a very unfavorable prognosis. ${ }^{6}$ In addition, both cases did not show the typical radiologic features of Paget's disease of bone at clinical presentation.

In summary, we describe two cases of central lowgrade osteosarcoma with unusual histologic features resembling Paget's disease of bone. These cases further expand the histologic spectrum of central low-grade osteosarcoma, and underscore the diagnostic difficulties in separating these neoplasms from benign bone diseases. In accordance with previous studies of larger series, inadequate treatment of these tumors may result in recurrence and eventually in dedifferentiation to high-grade osteosarcoma, with development of distant metastases. Recognition of this variant of central low-grade osteosarcoma is based on the aggressive radiologic appearance and on adequate tumor sampling for histologic examination.

\section{References}

1 Kurt AM, Unni KK, McLeod RA, et al. Low grade intraosseous osteosarcoma. Cancer 1990;65:1418-1428.

2 Bertoni F, Bacchini P, Fabbri N, et al. Osteosarcoma. Low-grade intraosseous-type osteosarcoma, histologically resembling parosteal osteosarcoma, fibrous displasia, and desmoplastic fibroma. Cancer 1993;71: 338-345.

3 Choong PF, Pritchard DJ, Rock MG, et al. Low-grade central osteogenic sarcoma. A long term follow-up of 20 patients. Clin Orthop 1996;322:198-206.

4 Okada K, Frassica FJ, Sim FH, et al. Parosteal osteosarcoma. A clinicopathological study. J Bone Joint Surg Am 1994;76:366-378.

5 Riminucci M, Liu B, Corsi A, et al. The histopathology of fibrous dysplasia of bone in patients with activating mutations of the Gs alpha gene: site-specific patterns and recurrent histological hallmarks. J Pathol 1999; 187:249-258.

6 Huvos AG, Butler A, Bretsky SS. Osteogenic sarcoma associated with Paget's disease of bone. A clinicopathologic study of 65 patients. Cancer 1983;52:1489-1495. 